\title{
Erratum: Evidence for a Phase Transition in Silicate Melt at Extreme Pressure and Temperature Conditions [Phys. Rev. Lett. 108, 065701 (2012)]
}

\author{
D. K. Spaulding, R. S. McWilliams, R. Jeanloz, J. H. Eggert, P. M. Celliers, D. G. Hicks, \\ G. W. Collins, and R. F. Smith
}

(Received 29 August 2017; published 29 September 2017)

DOI: 10.1103/PhysRevLett.119.139903

In our earlier publication we reported observations of anomalies in shock velocity and temperature along the shock Hugoniots of single-crystal and vitreous enstatite $\left(\mathrm{MgSiO}_{3}\right)$. Our sample description in Supplemental Material mistakenly suggests that all targets included a $2 \mu \mathrm{m}$ Au heat shield layer, but that is not the case. The significance of this detail is that a reverberation originating in a Au layer could have produced a discontinuity in shock velocity and temperature similar to that which we reported and interpreted as evidence for a transformation in liquid $\mathrm{MgSiO}_{3}$. In fact, the samples that showed a discontinuity in shock velocity and temperature did not include Au. We cannot, therefore, easily attribute our observations to an artifact of sample design. Otherwise identical experiments on other compositions did not show such anomalies.

A correct sample description should have made clear the following:

None of the five experiments that showed the reported anomaly in shock velocity and temperature included Au in the target package. A single sample used for one of our decaying shock experiments did include Au; however, there was no evidence of reverberation or discontinuity in shock velocity in that experiment, contrary to what is suggested by hydrodynamic simulations by ourselves and others. The decaying shock in this experiment appeared to originate at a pressure and temperature below the conditions of the apparent phase transition due to lower laser drive energy and showed a smooth decay in pressure, which was therefore consistent with our interpretation. All decaying shock experiments employed a $2 \mathrm{~ns}$ square pulse for the drive laser.

Of the ten data points reported for the equation of state of $\mathrm{MgSiO}_{3}$, eight of those experiments included $\mathrm{Au}$ and two did not. Those that included Au also did not show obvious signs of reverberation in either the quartz impedance standard or the sample. Equation of state experiments used either a 4 or a 2 ns square pulse for the drive laser.

While the change in target description would not, by itself, affect our central interpretations or conclusions, the source of the anomaly that we reported has come under scrutiny as more recent experiments have failed to observe the same effect $[1,2]$. We refer the reader to more recent experimental work for additional data and interpretations.

[1] R. M. Bolis, G. Morard, T. Vinci, A. Ravasio, E. Bambrink, M. Guarguaglini, M. Koenig, R. Musella, F. Remus, J. Bouchet, N. Ozaki, K. Miyanishi, T. Sekine, Y. Sakawa, T. Sano, R. Kodama, F. Guyot, and A. Benuzzi-Mounaix, Geophys. Res. Lett. 43, 9475 (2016).

[2] D. E. Fratanduono, R. G. Kraus, M. Millot, D. K. Spaulding, G. W. Collins, P. M. Celliers, and J. H. Eggert (to be published). 